\title{
Survival probabilities in bivariate risk models, with application to reinsurance
}

\author{
A. Castañer ${ }^{\mathrm{a}}$, M.M. Claramunt ${ }^{\mathrm{a}, *}$, C. Lefèvre ${ }^{\mathrm{b}}$ \\ ${ }^{a}$ Dept. Matemàtica Econòmica, Financera i Actuarial, Universitat de Barcelona, Av. Diagonal, 690, 08034 \\ Barcelona, Spain \\ ${ }^{b}$ Dept. Mathématique, Université Libre de Bruxelles, Boulevard du Triomphe, Campus de la Plaine C.P.210, \\ B-1050 Bruxelles, Belgium
}

\begin{abstract}
This paper deals with an insurance portfolio that covers two interdependent risks. The central model is a discrete-time bivariate risk process with independent claim increments. A continuoustime version of compound Poisson type is also examined. Our main purpose is to develop a numerical method for determining non-ruin probabilities over a finite-time horizon. The approach relies on, and exploits, the existence of a special algebraic structure of Appell type. Some applications in reinsurance to the joint risks of the cedent and the reinsurer are presented and discussed, under a stop-loss or excess of loss contract.
\end{abstract}

Keywords: multirisks model, discrete or continuous time, finite-time ruin probability, Appell algebraic structure, recursive methods, stop-loss and excess of loss reinsurance

2010 MSC: 91B30, 60G40

\section{Introduction}

Multivariate risk models aim to describe the evolution in time of an insurance portfolio that covers several interdependent risks. A typical situation is with certain damages or catastrophic events that cause losses in different branches. This is the case, for instance, in liability automobile insurance for material damage and bodily injury, or with natural catastrophe insurance for claims distributed by geographical zones. The presence of an economic environment or the application of common legal rules can also influence several business lines. Another important application is in reinsurance where the ceding and reinsurance companies are jointly liable for covering claim losses.

The analysis of multirisks models has received growing interest in recent years. Various assumptions have been considered on the claim arrival processes and the claim amount distributions. We refer e.g. to Avram et al. (2008), Cai and Li (2007), Chan et al. (2003), Denuit et al. (2007), Gong et al. (2012), Guo et al. (2007), Li et al. (2007), Picard et al. (2003a) and the references therein. For reinsurance applications, see e.g. Kaishev and Dimitrova (2006), Kaishev et al. (2008) and Dimitrova and Kaishev (2010).

The present work is concerned with the evaluation of non-ruin probabilities for a twodimensional risk process. The extension to multivariate cases is rather simple. We examine

\footnotetext{
${ }^{*}$ Corresponding author

Email addresses: acastaner@ub.edu (A. Castañer), mmclaramunt@ub.edu (M.M. Claramunt), clefevre@ulb.ac.be (C. Lefèvre)
} 
two different bivariate risk models. The central model, discussed in Section 2, is a discrete-time process with independent claim increments. As indicated e.g. in Li et al. (2009), a discrete-time modeling is often more appropriate in reality as an insurance usually makes balances at fixed periodic dates. Our model is a variant of the multivariate model studied in Picard et al. (2003a) and is an extension of the univariate model investigated in Castañer et al. (2011). Section 3 is concerned with a continuous-time version, of compound Poisson type, of the previous bivariate risk model. The mathematical analysis of that process is found to be slightly less intricate. An analogous multivariate model was studied e.g. in Cai and Li (2007), Chan et al. (2003). In Section 4, the discrete-time bivariate model is applied in reinsurance to a limited stop-loss or excess of loss contract. The survival probabilities of the cedent and/or reinusurer are computed and discussed with several numerical examples and problems.

Let $\left\{U_{1}(t), U_{2}(t)\right\}$ be the bivariate surplus process for the two risks. Its precise building, quite standard, will be given later on. Several definitions of ruin may be useful in multirisks management. Following e.g. Cai and Li (2007), we consider three possible definitions.

(i) Ruin occurs at time $T_{\text {or }}$ as soon as one of the two surpluses becomes negative. Thus, $T_{\text {or }}=\min \left(T_{1}, T_{2}\right)$ where $T_{i}=\inf \left\{t \geq 1: U_{i}(t)<0\right\}$ is the ruin time for risk $i$. So, the event $\left(T_{o r}>t\right)$ means that both surpluses remain always non-negative until $t$. This case is relevant for many applications, including reinsurance.

(ii) Ruin occurs at time $T_{\text {and }}$ when the two surpluses become negative, not necessarily at the same time. Thus, $T_{\text {and }}=\max \left(T_{1}, T_{2}\right)$. So, $\left(T_{\text {and }}>t\right)$ means that at least one of the two surpluses remains always non-negative until $t$. This case too may be of interest for practical problems.

(iii) Ruin occurs at time $T_{\text {sim }}$ when the two surpluses become negative simultaneously, i.e. at the same time. Thus, $T_{\text {sim }}=\inf \left\{t \geq 1: U_{1}(t)<0\right.$ and $\left.U_{2}(t)<0\right\}$. So, $\left(T_{\text {sim }}>t\right)$ means that at least one surplus is non-negative at each time until $t$. Note that a company is allowed here to function after its own ruin. This case seems to be less natural.

Our main purpose is to derive, for these three definitions of ruin, a simple formula that enables us to calculate survival probabilities over a finite-time horizon. The assumptions made on the premium incomes and claim processes will be rather general. As a consequence, the formulas obtained are not entirely explicit but require some calculations by recurrence. Our key result will be concerned with the ruin time $T_{o r}$. The cases of $T_{\text {and }}$ and $T_{\text {sim }}$ then follow easily. For both bivariate risk models under study, the recurrences involved rely on the existence of an algebraic structure of Appell type. That structure is polynomial for the continuous-time process, but somewhat different for the discrete-time process.

Appell and generalized Appell (Sheffer) polynomials are well-known in mathematics (e.g. Kaz'min, 2002). In insurance theory, their presence has been pointed out and exploited (to some extent) for various univariate risk models. The reader is referred e.g. to Dimitrova and Kaishev (2010), Ignatov and Kaishev (2000, 2004), Lefèvre and Loisel (2009), Lefèvre and Picard (2011), Picard and Lefèvre (1997), Picard et al. (2003b); see Picard et al. (2003a) for a multivariate risk model.

It is worth indicating that very few methods have been proposed so far to evaluate survival probabilities in a bivariate risk model. The approach developed here is feasible in practice, for both the discrete-time version and the continuous-time version of the model. Our experience has shown that computations are generally more efficient for the discrete-time model (which is also more relevant in insurance). 


\section{A discrete-time bivariate risk model}

The risk process of this Section is a discrete-time model for two dependent risks, labelled 1 and 2. It is a variant of the bivariate model discussed in Picard et al. (2003a); it generalizes the univariate model studied in Castañer et al. (2011). Let $t \in \mathbb{N}=\{0,1, \ldots\}$ be the time scale. The initial reserves are $u_{1}$ and $u_{2}$. During each period $(t-1, t], t \geq 1$, the company receives a total premium income of $c_{1, t}$ for risk 1 and $c_{2, t}$ for risk 2 . These premiums are collected at the beginning of the period, i.e. at time $(t-1)^{+}$, as often (other cases might be considered).

The total claim amounts during $(t-1, t], t \geq 1$, are non-negative random variables $X_{1, t}$ for risk 1 and $X_{2, t}$ for risk 2. These amounts are registered at the end of the period, i.e. at time $t$. Claim amounts have any continuous distribution, possibly time-dependent, but with also an atom at 0 to allow the possibility of no claim. The two risk processes have independent increments, i.e. the random vectors $\left(X_{1, t}, X_{2, t}\right), t \geq 1$, are independent. Of course, the amounts $X_{1, t}$ and $X_{2, t}$ are in general correlated as claims generate losses for both risks. Note that the claim arrival process in the course of time is not defined explicitly.

For each risk $i=1,2$, during the first $t$ periods, the aggregate premiums with the initial reserves are

$$
h_{i}(t)=u_{i}+c_{i, 1}+\ldots+c_{i, t},
$$

and the aggregate claim amounts to be covered are

$$
S_{i}(t)=\sum_{j=1}^{t} X_{i, j}, \quad t \geq 1 .
$$

Let $F_{t}\left(s_{1}, s_{2}\right)$ denote the joint distribution function of $\left[S_{1}(t), S_{2}(t)\right]$. We recall that, for instance, $F_{t}(\infty, 0)=P\left[S_{2}(t)=0\right]>0$ by hypothesis. The two surpluses $U_{i}(t)$ are then given by

$$
U_{i}(t)=h_{i}(t)-S_{i}(t), \quad t \geq 1 .
$$

We are going to propose a method to evaluate the non-ruin probabilities over any finite horizon, for the three definitions of ruin indicated in Section 1. The key case will be concerned with the definition (i).

Finite-time survival probabilities. Consider any time $t \geq 1$ and define

$$
\phi_{\text {or }}\left(t, x_{1}, x_{2}\right)=P\left[T_{\text {or }}>t, U_{1}(t) \geq x_{1}, U_{2}(t) \geq x_{2}\right] .
$$

This is the probability that ruin for each risk does not occur until $t$ and the two surpluses at $t$ are at least equal to $x_{1}$ and $x_{2}$. By construction, $0 \leq x_{1} \leq h_{1}(t), 0 \leq x_{2} \leq h_{2}(t)$. Note also that

$$
\phi_{\text {or }}(t, 0,0) \equiv \phi_{\text {or }}(t)=P\left(T_{\text {or }}>t\right)=P\left(T_{1}>t \text { and } T_{2}>t\right) .
$$

Now, let us define a family of integers $v\left(s_{1}, s_{2}\right)$, for any non-negative reals $s_{1}, s_{2}$, as follows. If $s_{1} \leq h_{1}(1)$ and $s_{2} \leq h_{2}(1)$, then $v\left(s_{1}, s_{2}\right)=0$; otherwise, put

$$
v\left(s_{1}, s_{2}\right)=\sup \left\{t \geq 1: h_{1}(t)<s_{1} \text { or } h_{2}(t)<s_{2}\right\} .
$$

In other words, $v\left(s_{1}, s_{2}\right)$ is the last time $t \geq 0$ when an aggregate claim amount of $s_{1}$ for risk 1 and $s_{2}$ for risk 2 would lead to ruin for at least one risk, if ever. This means that at time $v\left(s_{1}, s_{2}\right)^{+}$, the new premiums received would allow one to cover both claim amounts $s_{1}$ and $s_{2}$; see Figure 1, left side. 

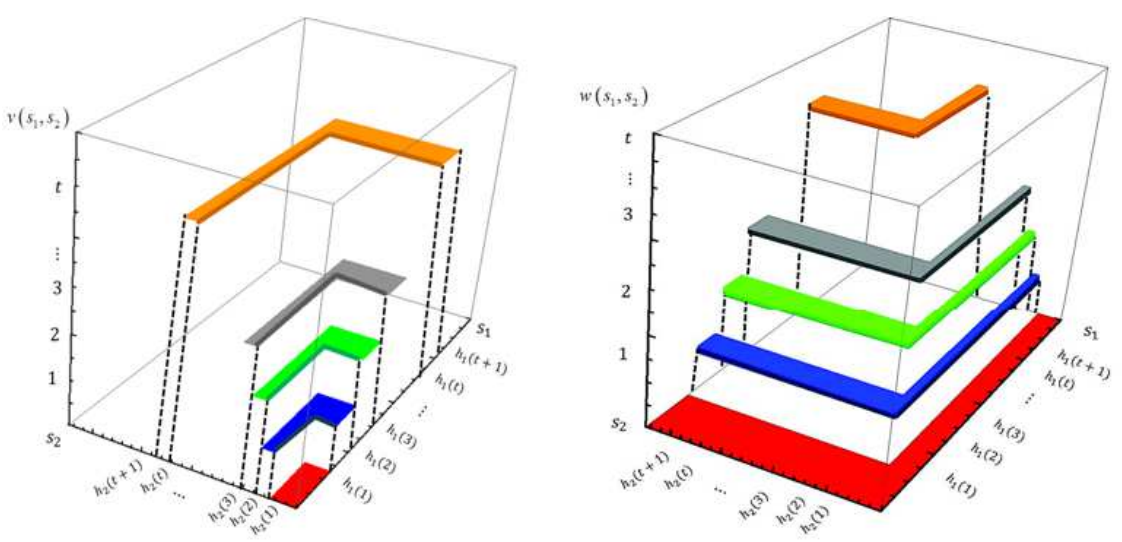

Figure 1: Graphs of $v\left(s_{1}, s_{2}\right)$ and $w\left(s_{1}, s_{2}\right)$ in function of $s_{1}, s_{2}$.

Proposition 2.1. For the definition (i),

$$
\begin{aligned}
& \phi_{\text {or }}\left(t, x_{1}, x_{2}\right)=F_{t}(0,0)+\int_{w_{1}=0}^{h_{1}(t)-x_{1}} b\left(w_{1}, 0\right) F_{t}\left[h_{1}(t)-x_{1}-w_{1}, 0\right] d w_{1} \\
& \quad+\int_{w_{2}=0}^{h_{2}(t)-x_{2}} b\left(0, w_{2}\right) F_{t}\left[0, h_{2}(t)-x_{2}-w_{2}\right] d w_{2} \\
& \quad+\int_{w_{1}=0}^{h_{1}(t)-x_{1}} \int_{w_{2}=0}^{h_{2}(t)-x_{2}} b\left(w_{1}, w_{2}\right) F_{t}\left[h_{1}(t)-x_{1}-w_{1}, h_{2}(t)-x_{2}-w_{2}\right] d w_{1} d w_{2},
\end{aligned}
$$

where $b\left(w_{1}, w_{2}\right)$ is a real function satisfying the equations

$$
\begin{aligned}
0 & =\int_{w_{1}=0}^{s_{1}} b\left(s_{1}-w_{1}, 0\right) d F_{v\left(s_{1}, 0\right)}\left(w_{1}, 0\right), \quad s_{1}>0 \\
0 & =\int_{w_{2}=0}^{s_{2}} b\left(0, s_{2}-w_{2}\right) d F_{v\left(0, s_{2}\right)}\left(0, w_{2}\right), \quad s_{2}>0, \\
0 & =\int_{w_{1}=0}^{s_{1}} \int_{w_{2}=0}^{s_{2}} b\left(s_{1}-w_{1}, s_{2}-w_{2}\right) d F_{v\left(s_{1}, s_{2}\right)}\left(w_{1}, w_{2}\right), \quad s_{1}, s_{2}>0 .
\end{aligned}
$$

Proof. Clearly,

$$
\begin{aligned}
\phi_{o r}\left(t, x_{1}, x_{2}\right) & =P\left[T_{o r}>t, S_{1}(t) \leq h_{1}(t)-x_{1}, S_{2}(t) \leq h_{2}(t)-x_{2}\right] \\
& =\int_{s_{1}=0^{-}}^{h_{1}(t)-x_{1}} \int_{s_{2}=0^{-}}^{h_{2}(t)-x_{2}} \phi_{s_{1}, s_{2}}(t) d s_{1} d s_{2},
\end{aligned}
$$

where

$$
\phi_{s_{1}, s_{2}}(t) d s_{1} d s_{2}=P\left[T_{o r}>t, s_{1}<S_{1}(t) \leq s_{1}+d s_{1}, s_{2}<S_{2}(t) \leq s_{2}+d s_{2}\right],
$$

and $0^{-}$indicates that there is a positive mass at 0 . We first observe that

$$
\phi_{0,0}(t)=P\left[T_{\text {or }}>t, S_{1}(t)=0, S_{2}(t)=0\right]=F_{t}(0,0) .
$$

Now, when $s_{1}+s_{2}>0$, let us consider the instant $v\left(s_{1}, s_{2}\right)$ introduced above. If $t \leq v\left(s_{1}, s_{2}\right)$, there is ruin at $t$ for at least one risk, hence $\phi_{s_{1}, s_{2}}(t)=0$. Suppose $t>v\left(s_{1}, s_{2}\right)$. By definition of $v\left(s_{1}, s_{2}\right)$, we see that ruin is impossible during the period $\left(v\left(s_{1}, s_{2}\right), t\right]$. Remember also that the claim process has independent increments. Thus, the event $\left[T_{o r}>t, s_{1}<S_{1}(t) \leq s_{1}+d s_{1}, s_{2}<\right.$ 
$\left.S_{2}(t) \leq s_{2}+d s_{2}\right]$ is equivalent to the two following events: for some $0^{-} \leq w_{1} \leq s_{1}$ and $0^{-} \leq w_{2} \leq s_{2}$

(a) $\left[T_{o r}>v\left(s_{1}, s_{2}\right), s_{1}-w_{1}<S_{1}\left(v\left(s_{1}, s_{2}\right)\right) \leq s_{1}-w_{1}+d s_{1}, s_{2}-w_{2}<S_{2}\left(v\left(s_{1}, s_{2}\right)\right) \leq s_{2}-w_{2}+d s_{2}\right]$, and

(b) $\left[S_{1}\left(v\left(s_{1}, s_{2}\right), t\right)=w_{1}, S_{2}\left(v\left(s_{1}, s_{2}\right), t\right)=w_{2}\right]$ where $S_{i}\left(v\left(s_{1}, s_{2}\right), t\right) \equiv S_{i}(t)-S_{i}\left(v\left(s_{1}, s_{2}\right)\right)$ counts the number of claims for risk $i$ during $\left(v\left(s_{1}, s_{2}\right), t\right]$. This yields

$$
\phi_{s_{1}, s_{2}}(t)=\int_{w_{1}=0^{-}}^{s_{1}} \int_{w_{2}=0^{-}}^{s_{2}} \phi_{s_{1}-w_{1}, s_{2}-w_{2}}\left(v\left(s_{1}, s_{2}\right)\right) d F_{\left[v\left(s_{1}, s_{2}\right), t\right]}\left(w_{1}, w_{2}\right),
$$

where $F_{\left[v\left(s_{1}, s_{2}\right), t\right]}\left(w_{1}, w_{2}\right)$ is the joint distribution function of $\left[S_{1}\left(v\left(s_{1}, s_{2}\right), t\right), S_{2}\left(v\left(s_{1}, s_{2}\right), t\right)\right]$.

Our next step is to prove that for $t \geq v\left(s_{1}, s_{2}\right), \phi_{s_{1}, s_{2}}(t)$ can be expressed as

$$
\begin{aligned}
& \phi_{s_{1}, 0}(t)=\int_{w_{1}=0}^{s_{1}} b\left(s_{1}-w_{1}, 0\right) d F_{t}\left(w_{1}, 0\right), \quad s_{1}>0, \\
& \phi_{0, s_{2}}(t)=\int_{w_{2}=0}^{s_{2}} b\left(0, s_{2}-w_{2}\right) d F_{t}\left(0, w_{2}\right), \quad s_{2}>0, \\
& \phi_{s_{1}, s_{2}}(t)=\int_{w_{1}=0}^{s_{1}} \int_{w_{2}=0}^{s_{2}} b\left(s_{1}-w_{1}, s_{2}-w_{2}\right) d F_{t}\left(w_{1}, w_{2}\right), \quad s_{1}, s_{2}>0,
\end{aligned}
$$

for appropriate real functions $b\left(s_{1}, s_{2}\right)$. Note here that, since $\phi_{s_{1}, s_{2}}\left(v\left(s_{1}, s_{2}\right)\right)=0$, the three relations (2.7) when $t=v\left(s_{1}, 0\right), v\left(0, s_{2}\right)$ and $v\left(s_{1}, s_{2}\right)$ provide the three announced integral relations (2.3). To begin with, risk increments being independent, we write that

$$
F_{t}\left(w_{1}, w_{2}\right)=\int_{y_{1}=0^{-}}^{w_{1}} \int_{y_{2}=0^{-}}^{w_{2}} F_{v\left(s_{1}, s_{2}\right)}\left(w_{1}-y_{1}, w_{2}-y_{2}\right) d F_{\left[v\left(s_{1}, s_{2}\right), t\right]}\left(y_{1}, y_{2}\right) .
$$

Now, we are going to show that substituting (2.8) in the right-hand side (r.h.s.) of (2.7) gives for $\phi_{s_{1}, s_{2}}(t)$ the desired formula (2.6). Consider the case $s_{1}, s_{2}>0$. The r.h.s. of (2.7) becomes

$$
\begin{aligned}
\int_{w_{1}=0}^{s_{1}} & \int_{w_{2}=0}^{s_{2}} b\left(s_{1}-w_{1}, s_{2}-w_{2}\right) \\
& \left\{\int_{y_{1}=0^{-}}^{w_{1}} \int_{y_{2}=0^{-}}^{w_{2}} d F_{v\left(s_{1}, s_{2}\right)}\left(w_{1}-y_{1}, w_{2}-y_{2}\right) d F_{\left[v\left(s_{1}, s_{2}\right), t\right]}\left(y_{1}, y_{2}\right)\right\} \\
= & \int_{y_{1}=0^{-}}^{s_{1}} \int_{y_{2}=0^{-}}^{s_{2}} d F_{\left[v\left(s_{1}, s_{2}\right), t\right]}\left(y_{1}, y_{2}\right) \\
& \left\{\int_{w_{1}=y_{1} \vee 0}^{s_{1}} \int_{w_{2}=y_{2} \vee 0}^{s_{2}} b\left(s_{1}-w_{1}, s_{2}-w_{2}\right) d F_{v\left(s_{1}, s_{2}\right)}\left(w_{1}-y_{1}, w_{2}-y_{2}\right)\right\} \\
= & \int_{y_{1}=0^{-}}^{s_{1}} \int_{y_{2}=0^{-}}^{s_{2}} d F_{\left[v\left(s_{1}, s_{2}\right), t\right]}\left(y_{1}, y_{2}\right) \\
& \left\{\int_{v_{1}=0}^{s_{1}-y_{1}} \int_{v_{2}=0}^{s_{2}-y_{2}} b\left(s_{1}-y_{1}-v_{1}, s_{2}-y_{2}-v_{2}\right) d F_{v\left(s_{1}, s_{2}\right)}\left(v_{1}, v_{2}\right)\right\} .
\end{aligned}
$$

By virtue of (2.7), the double integral $\{\ldots\}$ corresponds to $\phi_{s_{1}-y_{1}, s_{2}-y_{2}}\left(v\left(s_{1}, s_{2}\right)\right)$; note that (2.7) is applicable as $v\left(s_{1}, s_{2}\right) \geq v\left(s_{1}-y_{1}, s_{2}-y_{2}\right)$. Hence, (2.6) follows as desired. The two other cases $s_{1}>0, s_{2}=0$ and $s_{1}=0, s_{2}>0$ lead to (2.6) in the same way.

Finally, to get $\phi_{\text {or }}\left(t, x_{1}, x_{2}\right)$, it remains to insert the expression of $\phi_{s_{1}, s_{2}}(t)$ inside (2.4). Note that in (2.4), $s_{1} \leq h_{1}(t)-x_{1}$ and $s_{2} \leq h_{2}(t)-x_{2}$, hence $t>v\left(s_{1}, s_{2}\right)$. Thus, $\phi_{s_{1}, s_{2}}(t)$ in (2.4) is 
given by (2.5) and (2.7). First, consider the domain $s_{1}, s_{2}>0$. Its contribution to $\phi_{o r}\left(t, x_{1}, x_{2}\right)$ is

$$
\begin{aligned}
& \int_{s_{1}=0}^{h_{1}(t)-x_{1}} \int_{s_{2}=0}^{h_{2}(t)-x_{2}}\left\{\int_{w_{1}=0}^{s_{1}} \int_{w_{2}=0}^{s_{2}} b\left(s_{1}-w_{1}, s_{2}-w_{2}\right) d F_{t}\left(w_{1}, w_{2}\right)\right\} d s_{1} d s_{2} \\
& =\int_{w_{1}=0}^{h_{1}(t)-x_{1}} \int_{w_{2}=0}^{h_{2}(t)-x_{2}}\left\{\int_{s_{1}=w_{1}}^{h_{1}(t)-x_{1}} \int_{s_{2}=w_{2}}^{h_{2}(t)-x_{2}} b\left(s_{1}-w_{1}, s_{2}-w_{2}\right) d s_{1} d s_{2}\right\} d F_{t}\left(w_{1}, w_{2}\right) \\
& =\int_{w_{1}=0}^{h_{1}(t)-x_{1}} \int_{w_{2}=0}^{h_{2}(t)-x_{2}}\left\{\int_{v_{1}=0}^{h_{1}(t)-x_{1}-w_{1}} \int_{v_{2}=0}^{h_{2}(t)-x_{2}-w_{2}} b\left(v_{1}, v_{2}\right) d v_{1} d v_{2}\right\} d F_{t}\left(w_{1}, w_{2}\right) \\
& =\int_{v_{1}=0}^{h_{1}(t)-x_{1}} \int_{v_{2}=0}^{h_{2}(t)-x_{2}} b\left(v_{1}, v_{2}\right)\left\{\int_{w_{1}=0}^{h_{1}(t)-x_{1}-v_{1}} \int_{w_{2}=0}^{h_{2}(t)-x_{2}-v_{2}} d F_{t}\left(w_{1}, w_{2}\right)\right\} d v_{1} d v_{2},
\end{aligned}
$$

which provides the fourth term in the r.h.s. of (2.2). Similarly, the domains $s_{1}>0, s_{2}=0$ and $s_{1}=0, s_{2}>0$ bring the second and third terms of (2.2). By (2.5), the case $s_{1}=s_{2}=0$ give the first term. $\diamond$

Let us choose $x_{1}=x_{2}=0$, for instance. Formula (2.2) shows that $\phi_{o r}(t)$, the survival probability until time $t$, can be expressed as a sum of values of $F_{t}$, the distribution function of the aggregate claim amounts at time $t$ only. The coefficients in this sum, $b(.,$.$) , do not depend$ on $t$ and can be computed from the integral relations (2.3).

This representation is remarkable by its (relative) simplicity. It finds its origin in formula (2.6) where $\phi_{s_{1}, s_{2}}(t)$, function of $t$ with index $\left(s_{1}, s_{2}\right)$, is expanded in terms of the functions $\phi$ with previous indices $\left(s_{1}-w_{1}, s_{2}-w_{2}\right)$ and all evaluated at a same point $v\left(s_{1}, s_{2}\right)$. Such a formula is somewhat related with the theory of generalized Appell (Sheffer) polynomials (e.g. Niederhausen, 1988) and pseudopolynomials (e.g. Picard and Lefèvre, 1996). Roughly, let us say that a function $\phi_{s_{1}, s_{2}}(t)$ has a generalized Appell structure when a derivative type operator with indices $\left(w_{1}, w_{2}\right)$ applied to $\phi_{s_{1}, s_{2}}(t)$ gives $\phi_{s_{1}-w_{1}, s_{2}-w_{2}}(t)$. Then, (2.6) may be viewed as a Taylor type expansion of $\phi_{s_{1}, s_{2}}(t)$ at point $v\left(s_{1}, s_{2}\right)$ with respect to basic functions, $e_{w_{1}, w_{2}}\left[v\left(s_{1}, s_{2}\right), t\right]$, given by $d F_{\left[v\left(s_{1}, s_{2}\right), t\right]}\left(w_{1}, w_{2}\right)$. We refer the reader e.g. to Lefèvre and Picard (2006), formula (4.9), for a similar expansion in case of a single index.

The marginal survival probabilities are easily deduced. For instance, to obtain $\phi_{1}\left(t, X_{1}\right)=$ $P\left(T_{1}>t, U_{1}(t) \geq x_{1}\right)$, it suffices to put above $x_{2}=0$ and $X_{2, t}=0$ a.s. for all $t$. So, for each risk $i=1,2$, let $F_{i, t}(s)$ be the distribution function of $S_{i}(t)$, and define $v_{i}(s)=0$ if $s \leq u_{i}$, otherwise

$$
v_{i}(s)=\sup \left\{t \in \mathbb{N}: \mathrm{h}_{\mathrm{i}}(\mathrm{t})<\mathrm{s}_{\mathrm{i}}\right\} .
$$

We then get the following result (see also Castañer et al., 2011, Proposition 2.1).

Corollary 2.2. Marginally, for $i=1,2$,

$$
\phi_{i}\left(t, x_{i}\right)=F_{i, t}(0)+\int_{w=0}^{h_{i}(t)-x_{i}} b_{i}(w) F_{i, t}\left[h_{i}(t)-x_{i}-w\right] d w
$$

where $b_{i}(w)$ is a real function satisfying the equations

$$
0=\int_{w=0}^{s} b_{i}(s-w) d F_{v_{i}(s)}(w), \quad s>0 .
$$


Let us turn to the case of $T_{\text {and }}$. Define

$$
\phi_{\text {and }}\left(t, x_{1}, x_{2}\right)=P\left[\left(T_{1}>t, U_{1}(t) \geq x_{1}\right) \text { or }\left(T_{2}>t, U_{2}(t) \geq x_{2}\right)\right],
$$

i.e. the probability that at least one surplus remains non-negative until time $t$, with final reserves of minimal level $x_{i}$ for risk $i$. Note that

$$
\phi_{\text {and }}(t, 0,0) \equiv \phi_{\text {and }}(t)=P\left(T_{\text {and }}>t\right)=P\left(T_{1}>t \text { or } T_{2}>t\right) .
$$

The result below is straightforward.

Corollary 2.3. For the definition (ii),

$$
\phi_{\text {and }}\left(t, x_{1}, x_{2}\right)=\phi_{1}\left(t, x_{1}\right)+\phi_{2}\left(t, x_{2}\right)-\phi_{\text {or }}\left(t, x_{1}, x_{2}\right) .
$$

In the case of $T_{\text {sim }}$, the problem becomes a little more delicate. Consider

$$
\phi_{s i m ; s_{1}, s_{2}}(t) d s_{1} d s_{2}=P\left[T_{s i m}>t, s_{1}<S_{1}(t) \leq s_{1}+d s_{1}, s_{2}<S_{2}(t) \leq s_{2}+d s_{2}\right] .
$$

Remember that $\left(T_{\text {sim }}>t\right)$ means $\left[U_{1}(s)\right.$ or $U_{2}(s) \geq 0$ for all $\left.1 \leq s \leq t\right]$. So, instead of $v\left(s_{1}, s_{2}\right)$, we here introduce a family of integers $w\left(s_{1}, s_{2}\right)$ as follows: $w\left(s_{1}, s_{2}\right)=0$ if $s_{1} \leq h_{1}(1)$ or $s_{2} \leq h_{2}(1)$, otherwise

$$
w\left(s_{1}, s_{2}\right)=\sup \left\{t \geq 1: h_{1}(t)<s_{1} \text { and } h_{2}(t)<s_{2}\right\} .
$$

Thus, $w\left(s_{1}, s_{2}\right)$ is the last time when an aggregate claim amount of $s_{1}$ for risk 1 and $s_{2}$ for risk 2 would lead to ruin for both risks, if ever. This implies that at time $w\left(s_{1}, s_{2}\right)^{+}$, the new premiums received would allow us to cover at least one claim amount $s_{1}$ or $s_{2}$; see Figure 1, right side.

Now, let us adapt the argument followed for deriving $\phi_{s_{1}, s_{2}}(t)$ in the proof of Proposition 2.1. We then easily obtain that $\phi_{s i m ; s_{1}, s_{2}}(t)$ is given by an analogous expression.

Corollary 2.4. For the definition (iii), $\phi_{\text {sim } ; 0,0}(t)=F_{t}(0,0)$, and when $s_{1}+s_{2}>0, \phi_{\text {sim } ; s_{1}, s_{2}}(t)=$ 0 if $t \leq w\left(s_{1}, s_{2}\right)$ while if $t>w\left(s_{1}, s_{2}\right), \phi_{\text {sim; } s_{1}, s_{2}}(t)$ is provided by (2.7) with new coefficients $c\left(w_{1}, w_{2}\right)$ (in place of $b\left(w_{1}, w_{2}\right)$ ) that satisfy again the equations (2.3) but with $w\left(s_{1}, s_{2}\right)$ substituted for $v\left(s_{1}, s_{2}\right)$.

Clearly, $\phi_{\text {sim }}(t) \equiv P\left(T_{\text {sim }}>t\right)$ is given by

$$
\phi_{s i m}(t)=\int_{0^{-} \leq s_{1} \leq h_{1}(t) \text { or } 0^{-} \leq s_{2} \leq h_{2}(t)} \phi_{s i m ; s_{1}, s_{2}}(t) d s_{1} d s_{2},
$$

where $t>w\left(s_{1}, s_{2}\right)$ on the domain of integration. It then remains to insert $\phi_{s i m ; s_{1}, s_{2}}(t)$ provided by Corollary 2.4 inside (2.12). Unlike for $T_{o r}$, however, it is not possible to deduce a simplified formula for this integral. Observe that by the definitions of ruin,

$$
\phi_{\text {sim }}(t) \geq \phi_{\text {and }}(t) \geq \phi_{\text {or }}(t) .
$$

A related probability of special interest is

$$
\phi_{\text {sim }}\left(t, x_{1}, x_{2}\right)=P\left[T_{\text {sim }}>t, U_{1}(t) \geq x_{1}, U_{2}(t) \geq x_{2}\right],
$$

i.e. the survival probability until time $t$ with final reserves of minimal level $x_{i} \geq 0$ for risk $i=1,2$. It is directly seen that $\phi_{\text {sim }}\left(t, x_{1}, x_{2}\right)$ has exactly the same expression $(2.2)$ as $\phi_{\text {or }}\left(t, x_{1}, x_{2}\right)$ but 
with $c\left(w_{1}, w_{2}\right)$ substituted for $b\left(w_{1}, w_{2}\right)$. We note that $\phi_{\text {sim }}(t, 0,0)=P\left[T_{\text {sim }}>t-1, U_{1}(t) \geq\right.$ $\left.0, U_{2}(t) \geq 0\right]$, hence

$$
\phi_{\text {sim }}(t) \geq \phi_{\text {sim }}(t, 0,0) \geq \phi_{\text {or }}(t) .
$$

An aproximating computational method. In practice, the application of Proposition 2.1 requires a discretization of the claim amount distributions. Given a common span $d$, each continuous vector $\left(X_{1, t}, X_{2, t}\right), t \geq 1$, is approximated by some vector $\left(X_{1, t}^{(d)}, X_{2, t}^{(d)}\right)$ with values $\left(n_{1} d, n_{2} d\right)$ and probability mass function $p_{t}^{(d)}\left(n_{1}, n_{2}\right)=P\left(X_{1, t}^{(d)}=n_{1} d, X_{2, t}^{(d)}=n_{2} d\right), n_{1}, n_{2} \in \mathbb{N}$. Hereafter, we choose a stochastically lower bound for the continuous vector that is simply defined by

$$
p_{t}^{(d)}\left(n_{1}, n_{2}\right)=P\left[n_{1} d \leq X_{1, t} \leq\left(n_{1}+1\right) d, n_{2} d \leq X_{2, t} \leq\left(n_{2}+1\right) d\right] .
$$

Other discrete approximations might be considered, of course. Note that $p_{t}^{(d)}\left(n_{1}, n_{2}\right)>0$ in all points by construction. For $\left[S_{1}^{(d)}(t), S_{2}^{(d)}(t)\right]$, put $f_{t}^{(d)}\left(n_{1}, n_{2}\right)=P\left[S_{1}^{(d)}(t)=n_{1} d, S_{2}^{(d)}(t)=n_{2} d\right]$, with distribution function $F_{t}^{(d)}\left(n_{1}, n_{2}\right)$; for $S_{1}^{(d)}(t)$ only, an index 1 is added in the notation.

The ruin times for risks $i=1,2$ are $T_{i}^{(d)}=\inf \left\{t \geq 1: U_{i}^{(d)}(t)=h_{i}(t)-S_{i}^{(d)}(t)<0\right\}$. Let us focus on the probabilities

$$
\phi_{n_{1}, n_{2}}^{(d)}(t)=P\left[T_{o r}^{(d)}>t, S_{1}^{(d)}(t)=n_{1} d, S_{2}^{(d)}(t)=n_{2} d\right],
$$

which are discrete equivalents of $\phi_{s_{1}, s_{2}}(t)$ introduced in (2.4). Define $v^{(d)}\left(n_{1}, n_{2}\right)=0$ if $n_{1} d \leq$ $h_{1}(1)$ and $n_{2} d \leq h_{2}(1)$, otherwise $v^{(d)}\left(n_{1}, n_{2}\right)=\sup \left\{t \geq 1: h_{1}(t)<n_{1} d\right.$ or $\left.h_{2}(t)<n_{2} d\right\}$, similarly to $v\left(s_{1}, s_{2}\right)$ above. By definition, $\phi_{n_{1}, n_{2}}^{(d)}(t)=0$ if $t \leq v^{(d)}\left(n_{1}, n_{2}\right)$.

Corollary 2.5. For $t>v^{(d)}\left(n_{1}, n_{2}\right)$,

$$
\phi_{n_{1}, n_{2}}^{(d)}(t)=\sum_{k_{1}=0}^{n_{1}} \sum_{k_{2}=0}^{n_{2}} b^{(d)}\left(n_{1}-k_{1}, n_{2}-k_{2}\right) f_{t}^{(d)}\left(k_{1} d, k_{2} d\right),
$$

where $b^{(d)}(0,0)=1$ and the other coefficients $b^{(d)}\left(n_{1}, n_{2}\right)$ are derived recursively from

$$
0=\sum_{k_{1}=0}^{n_{1}} \sum_{k_{2}=0}^{n_{2}} b^{(d)}\left(n_{1}-k_{1}, n_{2}-k_{2}\right) f_{v^{(d)}\left(n_{1}, n_{2}\right)}^{(d)}\left(k_{1} d, k_{2} d\right), \quad n_{1}+n_{2} \geq 1 .
$$

Proof. We will argue as for Proposition 2.1. First, instead of (2.6), one finds here that

$$
\phi_{n_{1}, n_{2}}^{(d)}(t)=\sum_{k_{1}=0}^{n_{1}} \sum_{k_{2}=0}^{n_{2}} \phi_{n_{1}-k_{1}, n_{2}-k_{2}}^{(d)}\left(v^{(d)}\left(n_{1}, n_{2}\right)\right) f_{\left[v^{(d)}\left(n_{1}, n_{2}\right), t\right]}^{(d)}\left(k_{1}, k_{2}\right),
$$

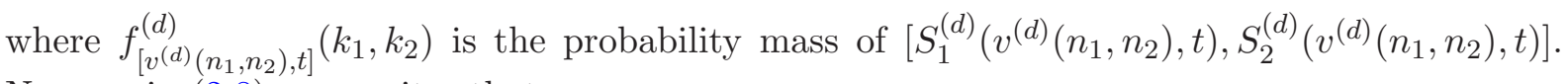
Now, as in (2.8), one writes that

$$
f_{t}^{(d)}\left(k_{1}, k_{2}\right)=\sum_{l_{1}=0}^{k_{1}} \sum_{l_{2}=0}^{k_{2}} f_{v^{(d)}\left(n_{1}, n_{2}\right)}^{(d)}\left(k_{1}-l_{1}, k_{2}-l_{2}\right) f_{\left[v^{(d)}\left(n_{1}, n_{2}\right), t\right]}^{(d)}\left(l_{1}, l_{2}\right) .
$$

Substituting (2.16) in the r.h.s. of (2.13) then gives for $\phi_{s_{1}, s_{2}}^{(d)}(t)$ the expected formula $(2.15)$. Furthermore, if $n_{1}=n_{2}=0, \phi_{0,0}^{(d)}(t)=P\left[S_{1}^{(d)}(t)=0, S_{2}^{(d)}(t)=0\right]$ by definition, hence $(2.13)$ 
implies $b^{(d)}(0,0)=1$. If $n_{1}+n_{2} \geq 1$, taking $t=v^{(d)}\left(n_{1}, n_{2}\right)$ gives $\phi_{n_{1}, n_{2}}^{(d)}\left(v^{(d)}\left(n_{1}, n_{2}\right)\right)=0$, hence (2.13) yields (2.14). $\diamond$

Most (non-)ruin probabilities of interest easily follow from this result. Some are given in the next corollary; the cases of $T_{\text {and }}^{(d)}$ and $T_{\text {sim }}^{(d)}$ are omitted for brevity. Let $\lfloor x\rfloor$ be the integer part of $x$.

\section{Corollary 2.6.}

$$
\begin{aligned}
& P\left(T_{\text {or }}^{(d)}>t\right)=\sum_{n_{1}=0}^{\left\lfloor h_{1}(t) / d\right\rfloor} \sum_{n_{2}=0}^{\left\lfloor h_{2}(t) / d\right\rfloor} b^{(d)}\left(n_{1}, n_{2}\right) F_{t}^{(d)}\left(\left\lfloor h_{1}(t) / d\right\rfloor-n_{1},\left\lfloor h_{2}(t) / d\right\rfloor-n_{2}\right) ; \\
& P\left(T_{1}^{(d)}>t\right)=\sum_{n=0}^{\left\lfloor h_{1}(t) / d\right\rfloor} b^{(d)}(n) F_{1, t}^{(d)}\left(\left\lfloor h_{1}(t) / d\right\rfloor-n\right) \text {, where } \\
& b^{(d)}(0)=1 \text { and } 0=\sum_{k=0}^{n} b^{(d)}(n) f_{1, v^{(d)}(n)}^{(d)}(k), \quad n \geq 1 ; \\
& P\left(T_{1}^{(d)}=t, T_{2}^{(d)}>t\right)=\sum_{n_{1}=0}^{\left\lfloor h_{1}(t) / d\right\rfloor} \sum_{n_{2}=0}^{\left\lfloor h_{2}(t) / d\right\rfloor} \phi_{n_{1}, n_{2}}^{(d)}(t-1) \\
& P\left[X_{1, t}^{(d)}>h_{1}(t)-n_{1} d, X_{2, t}^{(d)} \leq h_{2}(t)-n_{2} d\right] ; \\
& P\left(T_{1}^{(d)}=t, T_{2}^{(d)}=t+t^{\prime}\right)=\sum_{n_{1}=0}^{\left\lfloor h_{1}(t) / d\right\rfloor} \sum_{n_{2}=0}^{\left\lfloor h_{2}(t) / d\right\rfloor} \phi_{n_{1}, n_{2}}^{(d)}(t-1) \sum_{m_{2}=0}^{\left\lfloor h_{2}(t) / d\right\rfloor-n_{2}} \\
& P\left[X_{1, t}^{(d)}>h_{1}(t)-n_{1} d, X_{2, t}^{(d)}=m_{2} d\right] P\left[T_{2}^{(d)}=t^{\prime} \mid t,\left(n_{2}+m_{2}\right) d\right], \quad t^{\prime} \geq 1,
\end{aligned}
$$

where $P\left[T_{2}^{(d)}=t^{\prime} \mid t,\left(n_{2}+m_{2}\right)\right]$ is the ruin probability at time $t+t^{\prime}$ for the single risk 2 when starting at time $t$ with initial reserves $h_{2}(t)-\left(n_{2}+m_{2}\right) d$.

Proof. For (2.17), starting with

$$
P\left(T_{\text {or }}^{(d)}>t\right)=\sum_{n_{1}=0}^{\left\lfloor h_{1}(t) / d\right\rfloor} \sum_{n_{2}=0}^{\left\lfloor h_{2}(t) / d\right\rfloor} \phi_{n_{1}, n_{2}}^{(d)}(t),
$$

one may insert (2.13) in the r.h.s. (because $t>v^{(d)}\left(n_{1}, n_{2}\right)$ ) and it then suffices to permute the sums involved. The marginal probability (2.18) follows as in Corollary 2.2. The two other formulas are direct too. $\diamond$

\section{A compound Poisson bivariate risk model}

This Section is concerned with a continuous-time version of the previous bivariate risk model. It is analogous to the model studied e.g. in Cai and Li (2007), Chan et al. (2003); it generalizes the classical (univariate) compound Poisson risk model. Let $t \in \mathbb{R}^{+}$. For each risk $i=1,2$, the aggregate premiums up to time $t$ plus the initial reserves are given by a non-decreasing function $h_{i}(t)$.

Claim arrivals are generated by a Poisson process $N(t), t \geq 0$, with parameter $\lambda$. For risk $i$, the consecutive claim amounts form a sequence of random variables $X_{i, j}, j \geq 1$, with any 
continuous distribution, possibly non-stationary. The variables $X_{1, j}$ and $X_{2, j}$ may be interdependent. Denote the sum of the first $n$ claim amounts by $S_{i, n}=X_{i, 1}+\ldots+X_{i, n}, n \geq 1$; put $S_{i, 0}=0$. The two surpluses are then given by

$$
U_{i}(t)=h_{i}(t)-S_{i, N(t)}, \quad t \geq 0 .
$$

Here too, we aim to obtain a formula allowing us to compute the non-ruin probabilities over a finite horizon.

Finite-time survival probabilities. For any time $t>0$, define as before $\phi_{o r}\left(t, x_{1}, x_{2}\right)=$ $P\left[T_{o r}>t, U_{1}(t) \geq x_{1}, U_{2}(t) \geq x_{2}\right]$. The derivation of this probability will differ from the discrete-time case because claims here occur at random instants instead of periodic times. The fact that these instants are generated by a Poisson process will enable us to point out a reinforced algebraic structure in terms of classical Appell polynomials.

The existence of such an Appell structure has been shown, in a context of reinsurance, in Kaishev and Dimitrova (2006), Theorems 1 and 2. The framework below is more general, and our approach is much more direct as it exploits interesting properties of these polynomials. This approach is an adaptation of a method of proof developed recently for a single risk in Lefèvre and Picard (2011), Section 4. First, we will point out why Appell polynomials play a natural role for deriving the survival probabilities in the present risk model. Then, we will underline the computational advantage of the Appell structure.

Let us recall the definition of Appell polynomials (see e.g. Picard and Lefèvre, 1996). Consider a given real sequence $V=\left\{v_{j}, j \geq 0\right\}$, increasing for instance. To $V$ is attached a unique family of Appell polynomials $A_{n}(x \mid V), n \geq 0$, of degree $n$ in $x$ where $A_{0}=1$ and the other $A_{n}$ 's are constructed recursively through the key identity

$$
\frac{d A_{n}(x \mid V)}{d x}=A_{n-1}(x \mid V), \text { with } A_{n}\left(v_{n-1} \mid V\right)=0, \quad n \geq 1 .
$$

Thus, an integral representation is

$$
A_{n}(x \mid V)=\int_{y_{n-1}=v_{n-1}}^{x} d y_{n-1} \int_{y_{n-2}=v_{n-2}}^{y_{n-1}} d y_{n-2} \ldots \int_{y_{0}=v_{0}}^{y_{1}} d y_{0}, \quad n \geq 1 .
$$

Note that $A_{n}$ depends on $V$ only through the first $n$ terms $v_{0}, \ldots, v_{n-1}$.

Now, define a function $v\left(s_{1}, s_{2}\right)$, for $s_{1}, s_{2} \geq 0$, as in Section 2, i.e. $v\left(s_{1}, s_{2}\right)=0$ if $s_{1} \leq h_{1}(0)$ and $s_{2} \leq h_{2}(0)$, otherwise

$$
v\left(s_{1}, s_{2}\right)=\sup \left\{t>0: h_{1}(t)<s_{1} \text { or } h_{2}(t)<s_{2}\right\} .
$$

Let $I[B]$ be the indicator of an event $B$.

Proposition 3.1. For the definition (i),

$$
\begin{aligned}
\phi_{\text {or }}\left(t, x_{1}, x_{2}\right)=E\left\{N(t) ! A_{N(t)}[\right. & \left.1 \mid v\left(S_{1,1}, S_{2,1}\right) / t, \ldots, v\left(S_{1, N(t)}, S_{2, N(t)}\right) / t\right] \\
I & {\left.\left[S_{1, N(t)} \leq h_{1}(t)-x_{1}, S_{2, N(t)} \leq h_{2}(t)-x_{2}\right]\right\} . }
\end{aligned}
$$

Proof. To start, we condition on the number of claims in $(0, t)$ and the cumulated claim amounts for the two risks. For $N(t)=n \geq 1$, define the conditional probabilities

$$
\begin{aligned}
& \phi_{n,\left(s_{1,1}, s_{2,1}\right), \ldots,\left(s_{1, n}, s_{2, n}\right)}^{c}(t)= \\
& \quad P\left[T_{o r}>t \mid N(t)=n,\left(S_{1,1}=s_{1,1}, S_{2,1}=s_{2,1}\right), \ldots,\left(S_{1, n}=s_{1, n}, S_{2, n}=s_{2, n}\right)\right],
\end{aligned}
$$


and let $F_{n}\left[\left(s_{1,1}, s_{2,1}\right), \ldots,\left(s_{1, n}, s_{2, n}\right)\right]$ be the joint distribution function of the first $n$ claim sums. Then,

$$
\phi_{o r}\left(t, x_{1}, x_{2}\right)=\sum_{n=0}^{\infty} P[N(t)=n] P\left[T_{o r}>t, S_{i, n} \leq h_{i}(t)-x_{i} \text { for } i=1,2 \mid N(t)=n\right],
$$

in which $P[\ldots \mid N(t)=0] \equiv 1$, and $P[\ldots \mid N(t)=n], n \geq 1$, is given by

$$
\int_{\mathcal{D}_{n, t, x_{1}, x_{2}}} \phi_{n,\left(s_{1,1}, s_{2,1}\right), \ldots,\left(s_{1, n}, s_{2, n}\right)}^{c}(t) d F_{n}\left[\left(s_{1,1}, s_{2,1}\right), \ldots,\left(s_{1, n}, s_{2, n}\right)\right],
$$

where $\mathcal{D}_{n, t, x_{1}, x_{2}}$ is the set $\left\{0 \leq s_{i, 1} \leq \ldots \leq s_{i, n} \leq h_{i}(t)-x_{i}\right.$ for $\left.i=1,2\right\}$.

Now, given $n \geq 1$ claims during $(0, t)$, let $\tau_{1}<\ldots<\tau_{n}$ be their arrival times. Consider, for instance, the first claim, of amount $\left(s_{1,1}, s_{2,1}\right)$. That claim does not cause ruin for risk 1 if $h_{1}\left(\tau_{1}\right) \geq s_{1,1}$ and for risk 2 if $h_{2}\left(\tau_{1}\right) \geq s_{2,1}$, i.e. if $\tau_{1}>v\left(s_{1,1}, s_{2,1}\right)$. A similar argument can be made for the next claims. Remember also that the claim times and amounts are independent. Thus, the conditional probability $\phi^{c}$ in (3.6) can be expressed as

$$
P\left[\tau_{1}>v\left(s_{1,1}, s_{2,1}\right), \ldots, \tau_{n}>v\left(s_{1, n}, s_{2, n}\right) \mid N(t)=n\right] .
$$

It is well-known that for a Poisson process, if $n$ events occur during $(0, t)$, the $n$ consecutive arrival times are distributed as the order statistics, $\left[U_{1: n}(0, t), \ldots, U_{n: n}(0, t)\right]$, of a sample of $n$ independent uniform $(0, t)$ random variables. So, $(3.7)$ can be rewritten as

$$
P\left[U_{1: n}(0,1)>v\left(s_{1,1}, s_{2,1}\right) / t, \ldots, U_{n: n}(0,1)>v\left(s_{1, n}, s_{2, n}\right) / t\right] .
$$

From (3.3), one sees that the right-tail distribution of the vector $\left[U_{1: n}(0,1), \ldots, U_{n: n}(0,1)\right]$ corresponds to a particular Appell polynomial. More precisely, for $0 \leq v_{1} \leq \ldots \leq v_{n} \leq 1$,

$$
P\left[U_{1: n}(0,1)>v_{1}, \ldots, U_{n: n}(0,1)>v_{n}\right]=n ! A_{n}\left(1 \mid v_{1}, \ldots, v_{n}\right) ;
$$

see also e.g. Denuit et al. (2003). From (3.8), we thus obtain that

$$
\phi_{n,\left(s_{1,1}, s_{2,1}\right), \ldots,\left(s_{1, n}, s_{2, n}\right)}^{c}(t)=n ! A_{n}\left[1 \mid v\left(s_{1,1}, s_{2,1}\right) / t, \ldots, v\left(s_{1, n}, s_{2, n}\right) / t\right], \quad n \geq 1 .
$$

Combining (3.5), (3.6), (3.9) and $A_{0}=1$ then provides formula (3.4). $\diamond$

It is worth mentioning that the structure of formula (3.4) is preserved if, instead of a Poisson process, $N(t), t \geq 0$, is a linear death process or a linear birth process with immigration. By adapting Lefèvre and Picard (2011), one can show that the only change in (3.4) is to write this time $A_{N(t)}\left[1 \mid H_{t}\left(v\left(S_{1,1}, S_{2,1}\right)\right), \ldots, H_{t}\left(v\left(S_{1, N(t)}, S_{2, N(t)}\right)\right)\right]$, where $H_{t}$ is some known function (omitted here). Furthermore, it is directly seen that the same result (3.4) remains true even if the claim amounts are correlated in the course of time. This remark is not valid for the model of Section 2.

We now deduce from (3.4) a more explicit and easily tractable formula for $\phi_{o r}\left(t, x_{1}, x_{2}\right)$. It will also enlighten us on the effects of the claim number and severities.

\section{Corollary 3.2.}

$$
\begin{gathered}
\phi_{\text {or }}\left(t, x_{1}, x_{2}\right)=\sum_{j=0}^{\infty} \sum_{k=0}^{j} m_{k, j}(t) E\left\{A_{k}\left[0 \mid v\left(S_{1,1}, S_{2,1}\right) / t, \ldots, v\left(S_{1, k}, S_{2, k}\right) / t\right]\right. \\
\left.I\left[S_{i, j} \leq h_{i}(t)-x_{i} \text { for } i=1,2, S_{i, j+1}>h_{i}(t)-x_{i} \text { for } i=1 \text { or } 2\right]\right\},
\end{gathered}
$$

where $m_{k, j}(t) \equiv \sum_{n=k}^{j} n(n-1) \ldots(n-k+1) P[N(t)=n]$. 
Proof. By (3.4),

$$
\phi_{o r}\left(t, x_{1}, x_{2}\right)=\sum_{n=0}^{\infty} P[N(t)=n] n ! E\left\{A_{n}\left[1 \mid v\left(S_{1,1}, S_{2,1}\right) / t, \ldots, v\left(S_{1, n}, S_{2, n}\right) / t\right] I\left[B_{n}(t)\right]\right\},
$$

where $B_{n}(t)$ denotes the event $\left[S_{i, n} \leq h_{i}(t)-x_{i}\right.$ for $\left.i=1,2\right]$. Consider the first time after $n$, $j+1$ say, when $B_{j+1}(t)$ is not true, i.e. $\left[S_{i, j+1}>h_{i}(t)-x_{i}\right.$ for $i=1$ or 2]. Clearly, $I\left[B_{n}(t)\right]=$ $\sum_{j=n}^{\infty} I\left[C_{j}(t)\right]$ where $C_{j}(t)$ is the event $\left[S_{i, j} \leq h_{i}(t)-x_{i}\right.$ for $i=1,2, S_{i, j+1}>h_{i}(t)-x_{i}$ for $i=$ 1 or 2]. Inserting this and permuting the two sums, we obtain

$$
\phi_{\text {or }}\left(t, x_{1}, x_{2}\right)=\sum_{j=0}^{\infty} \sum_{n=0}^{j} P[N(t)=n] n ! E\left\{A_{n}\left[1 \mid v\left(S_{1,1}, S_{2,1}\right) / t, \ldots, v\left(S_{1, n}, S_{2, n}\right) / t\right] I\left[C_{j}(t)\right]\right\} .
$$

Observe that by (3.2), the Taylor expansion of a polynomial $A_{n}(x \mid V)$ is given by

$$
A_{n}(x \mid V)=\sum_{k=0}^{n} A_{k}(0 \mid V) x^{n-k} /(n-k) !
$$

Let us apply (3.12) to $A_{n}[1 \mid \ldots]$ in (3.11). After permutation of two sums, we deduce the desired formula (3.10).

Note that in (3.10), the roles of $N(t)$ and the claim sums are dissociated to some extent. For instance, changing the claim amounts will only affect the factors $E\{\ldots\}$.

To apply (3.10), it is first necessary to determine the terms $A_{k}[0 \mid \ldots]$. The integral representation (3.3) is not convenient for that. A more efficient method consists in using the following recursion: $A_{0}=1$ and

$$
0=\sum_{k=0}^{n} A_{k}(0 \mid V) v_{n-1}^{n-k} /(n-k) !, \quad n \geq 1 .
$$

which is obtained from Taylor's formula (3.12) for $x=v_{n-1}$, with (3.2).

Formulas like (3.4) and (3.10) for $\phi_{\text {or }}(t)$ have been derived and implemented for various reinsurance problems in Dimitrova and Kaishev (2010), Kaishev and Dimitrova (2006), Kaishev et al. (2008). Such computations are indeed feasible but may be rather long, even for a single risk. From our experience, it seems to us preferable, for the numerical efficiency, to work with the discrete-time risk model of Section 2. Moreover, as already indicated, a discrete-time scale is generally more appropriate in insurance. This will be the choice made in the next Section for some applications to reinsurance.

To close, we mention that the probabilities $\phi_{i}\left(t, x_{i}\right)$ for risk $i=1,2$, and $\phi_{\text {and }}\left(t, x_{1}, x_{2}\right)$ in case of definition (ii) follow directly from (3.4) and (3.10) (as in Corollaries 2.3 and 2.4). For the definition (iii), one gets, in place of (2.12),

$$
\begin{array}{r}
\phi_{\text {sim }}(t)=E\left\{N(t) ! A_{N(t)}\left[1 \mid w\left(S_{1,1}, S_{2,1}\right) / t, \ldots, w\left(S_{1, N(t)}, S_{2, N(t)}\right) / t\right]\right. \\
\left.I\left[S_{1, N(t)} \leq h_{1}(t) \text { or } S_{2, N(t)} \leq h_{2}(t)\right]\right\},
\end{array}
$$

where $w\left(s_{1}, s_{2}\right)=0$ if $s_{1} \leq h_{1}(0)$ or $s_{2} \leq h_{2}(0)$, otherwise

$$
w\left(s_{1}, s_{2}\right)=\sup \left\{t>0: h_{1}(t)<s_{1} \text { and } h_{2}(t)<s_{2}\right\} .
$$




\section{Some applications to reinsurance}

In this Section, we use the bivariate risk model in a context of reinsurance to describe the joint surplus processes of the ceding and reinsurance companies. The time scale is discrete, so that we will apply the methodology developed in Section 2.

Two types of reinsurance are considered: a limited stop-loss contract and an excess of loss contract. For actuarial questions and optimality problems in reinsurance, see e.g. the books by Goovaerts et al. (1990), Kaas et al. (2008) and the papers by Centeno and Simões (2009), Rytgaard (2004), with the references therein. Most often, attention is focused on the interest of solely the cedent company. In reality, however, the (contradicting) interests of both the cedent and the reinsurer have to be taken into account. This is an approach proposed recently in e.g. Dimitrova and Kaishev (2010), Kaishev and Dimitrova (2006), Kaishev et al. (2008), under an excess of loss reinsurance contract with a retention and a limiting level. Hereafter, we will first examine a stop-loss contract with similar characteristics and then, more briefly, again an excess of loss contract.

\subsection{Stop-loss reinsurance}

We start by formulating a reinsurance model for a limited stop-loss contract. For simplicity, we only consider the stationary case where all the model components remain constant over time. Let $U_{1}(t)$ and $U_{2}(t)$ be the surpluses of the cedent and the reinsurer, respectively. The total claim amounts during the successive periods $(t-1, t], t \geq 1$, are i.i.d. random variables $X_{t}$, distributed as $X$. To each amount $X_{t}$, both companies agree to fix a retention level $r>0$ and a limiting level $m>r$. Thus, the risk really covered by the cedent is

$$
X_{1, t}=X_{t} I\left(X_{t} \leq r\right)+r I\left[r<X_{t}<m\right]+\left[X_{t}-(m-r)\right] I\left(X_{t} \geq m\right),
$$

distributed as $X_{1}$, and the risk for the reinsurer is $X_{2, t}=X_{t}-X_{1, t}$, distributed as $X_{2}$. Note that the claim amounts $X_{1, t}$ and $X_{2, t}$ are here dependent through the global claim amount $X_{t}$.

Now, we assume that both companies apply the expected value principle to determine their premium levels. Let $c_{1}$ and $c_{2}$ be the premiums asked by the cedent and the reinsurer, given their safety loading factors $\theta_{1}$ and $\theta_{2}$. Then, $c_{1}=E(X)\left(1+\theta_{1}\right)-c_{2}$, and $c_{2}=E\left(X_{2}\right)\left(1+\theta_{2}\right)$. One easily sees that $E\left(X_{2}\right)=\pi_{X}(r)-\pi_{X}(m)$, where $\pi_{X}(r)=\int_{r}^{\infty}(x-r) d F_{X}(x)$ is the standard stop-loss premium for a risk $X$ with deductible $r$. An explicit expression for $\pi_{X}(r)$ is available only for special distributions of $X$ (such as exponential, lognormal and gamma). If $X$ has a compound distribution and Panjer's recursion may be applied, the calculation of $\pi_{X}(r)$ is also an easy task.

To estimate the distribution of $X$, we use a standard approximation method by a translated gamma distribution (as e.g. in Bowers et al., 1997). Specifically, let $G$ be a gamma random variable with (positive) parameters $(\alpha, \beta)$, i.e. with distribution function $P(G \leq x) \equiv G(x \mid \alpha, \beta)$ defined by

$$
G(x \mid \alpha, \beta)=\frac{\beta^{\alpha}}{\Gamma(\alpha)} \int_{0}^{x} y^{\alpha-1} e^{-\beta y} d y, \quad x>0 .
$$

The claim amount $X$ is approximated by a random variable $G+x_{0}$ such that the first three central moments coincide. Putting $\mu_{i}(X)=E(X-E(X))^{i}$, this yields

$$
\alpha=4 \mu_{2}^{3}(X) / \mu_{3}^{2}(X), \quad \beta=2 \mu_{2}(X) / \mu_{3}(X), \quad x_{0}=E(X)-\alpha / \beta .
$$

For such a distribution (see e.g. Kaas, 1993),

$$
\pi_{X}(r)=(\alpha / \beta)\left[1-G\left(r-x_{0} \mid \alpha+1, \beta\right)\right]-\left(r-x_{0}\right)\left[1-G\left(r-x_{0} \mid \alpha, \beta\right] .\right.
$$


Since $X$ is continuous, it will be discretised, as in Section 2, using a span of $d$. This provides a random variable $X^{(d)}$ with values $n d$ and probability masses $p^{(d)}(n) \equiv P\left(X^{(d)}=n d\right)=P[n d \leq$ $X \leq(n+1) d], n \in \mathbb{N}$. Then, $\left(X_{1}, X_{2}\right)$ is approximated by $\left(X_{1}^{(d)}, X_{2}^{(d)}\right)$ with values $\left(n_{1} d, n_{2} d\right)$ and probability masses $p^{(d)}\left(n_{1}, n_{2}\right) \equiv P\left(X_{1}^{(d)}=n_{1} d, X_{2}^{(d)}=n_{2} d\right)$ given by

$$
p^{(d)}\left(n_{1}, n_{2}\right)= \begin{cases}p^{(d)}\left(n_{1}\right), & \text { for } 0 \leq n_{1} \leq\lfloor r / d\rfloor, n_{2}=0, \\ p^{(d)}\left(\lfloor r / d\rfloor+n_{2}\right), & \text { for } n_{1}=\lfloor r / d\rfloor, 1 \leq n_{2} \leq\lfloor(m-r) / d\rfloor, \\ p^{(d)}\left(n_{1}+\lfloor(m-r) / d\rfloor\right), & \text { for } n_{1} \geq\lfloor r / d\rfloor+1, n_{2}=\lfloor(m-r) / d\rfloor .\end{cases}
$$

For illustration, suppose that $X$ has a translated gamma distribution with parameters $(\alpha=$ $\left.8 / 9, \beta=2 / 3, x_{0}=-1 / 3\right)$. Note that this distribution could provide an approximation to a compound Poisson distribution with parameter 1 and i.i.d. exponential individual claims of mean 1. Consider a stop-loss reinsurance contract with $r=0.8$ and $m=1.5$. Thus, $E(X)=1$ and $E\left(X_{2}\right)=0.228983$. We choose $\theta_{1}=0.05$ and $\theta_{2}=0.1$ for the loading factors, and different levels for the initial reserves $u_{1}, u_{2}$ of the cedent and the reinsurer.

(Non-)ruin probabilities. Table 1 gives the probabilities that before (or at) time $t=5$, ruin occurs for the cedent, the reinsurer, one of them at least or both companies. These results were obtained using formulas $(2.18),(2.17),(2.11)$, with a span $d=0.01$. Ruin probabilities for the cedent, the reinsurer and one of them are plotted in Figure 2. Of course, ruin risks become smaller with higher initial reserves. By this example, it seems that an increase of $u_{2}$, the initial reserves of the reinsurer, has a bigger effect on the ruin probabilities of at least one or both companies than the same increase of $u_{1}$, the initial reserves of the cedent. 
Table 1: Ruin probabilities until $t=5$ for the cedent, the reinsurer, one of them or both, in function of $\left(u_{1}, u_{2}\right)$ $\left(r=0.8, m=1.5, \theta_{1}=0.05, \theta_{2}=0.1\right.$ and $\left.d=0.01\right)$.

\begin{tabular}{|c|c|c|c|c|c|c|}
\hline$u_{1} \backslash u_{2}$ & & 0 & 0.25 & 0.5 & 0.75 & 1 \\
\hline \multirow{4}{*}{0} & $P\left(T_{1}^{(d)} \leq 5\right)$ & 0.6577 & 0.6577 & 0.6577 & 0.6577 & 0.6577 \\
\hline & $P\left(T_{2}^{(d)} \leq 5\right)$ & 0.6566 & 0.4887 & 0.2871 & 0.1882 & 0.0891 \\
\hline & $P\left(T_{o r}^{(d)} \leq 5\right)$ & 0.7362 & 0.6825 & 0.6663 & 0.6602 & 0.6581 \\
\hline & $P\left(T_{\text {and }}^{(d)} \leq 5\right)$ & 0.5781 & 0.4639 & 0.2785 & 0.1857 & 0.0888 \\
\hline \multirow{4}{*}{0.1} & $P\left(T_{1}^{(d)} \leq 5\right)$ & 0.5596 & 0.5596 & 0.5596 & 0.5596 & 0.5596 \\
\hline & $P\left(T_{2}^{(d)} \leq 5\right)$ & 0.6566 & 0.4887 & 0.2871 & 0.1882 & 0.0891 \\
\hline & $P\left(T_{o r}^{(d)} \leq 5\right)$ & 0.7065 & 0.6225 & 0.5785 & 0.5654 & 0.5607 \\
\hline & $P\left(T_{\text {and }}^{(d)} \leq 5\right)$ & 0.5097 & 0.4259 & 0.2682 & 0.1825 & 0.0881 \\
\hline \multirow{4}{*}{0.25} & $P\left(T_{1}^{(d)} \leq 5\right)$ & 0.5269 & 0.5269 & 0.5269 & 0.5269 & 0.5269 \\
\hline & $P\left(T_{2}^{(d)} \leq 5\right)$ & 0.6566 & 0.4887 & 0.2871 & 0.1882 & 0.0891 \\
\hline & $P\left(T_{o r}^{(d)} \leq 5\right)$ & 0.7016 & 0.6105 & 0.5540 & 0.5361 & 0.5288 \\
\hline & $P\left(T_{\text {and }}^{(d)} \leq 5\right)$ & 0.4819 & 0.4051 & 0.2600 & 0.1790 & 0.0872 \\
\hline \multirow{4}{*}{0.5} & $P\left(T_{1}^{(d)} \leq 5\right)$ & 0.4760 & 0.4760 & 0.4760 & 0.4760 & 0.4760 \\
\hline & $P\left(T_{2}^{(d)} \leq 5\right)$ & 0.6566 & 0.4887 & 0.2871 & 0.1882 & 0.0891 \\
\hline & $P\left(T_{o r}^{(d)} \leq 5\right)$ & 0.6945 & 0.5929 & 0.5175 & 0.4921 & 0.4798 \\
\hline & $P\left(T_{\text {and }}^{(d)} \leq 5\right)$ & 0.4381 & 0.3718 & 0.2455 & 0.1721 & 0.0853 \\
\hline \multirow{4}{*}{0.75} & $P\left(T_{1}^{(d)} \leq 5\right)$ & 0.4294 & 0.4294 & 0.4294 & 0.4294 & 0.4294 \\
\hline & $P\left(T_{2}^{(d)} \leq 5\right)$ & 0.6566 & 0.4887 & 0.2871 & 0.1882 & 0.0891 \\
\hline & $P\left(T_{o r}^{(d)} \leq 5\right)$ & 0.6885 & 0.5778 & 0.4860 & 0.4533 & 0.4357 \\
\hline & $P\left(T_{\text {and }}^{(d)} \leq 5\right)$ & 0.3975 & 0.3403 & 0.2304 & 0.1643 & 0.0828 \\
\hline \multirow{4}{*}{1} & $P\left(T_{1}^{(d)} \leq 5\right)$ & 0.3868 & 0.3868 & 0.3868 & 0.3868 & 0.3868 \\
\hline & $P\left(T_{2}^{(d)} \leq 5\right)$ & 0.6566 & 0.4887 & 0.2871 & 0.1882 & 0.0891 \\
\hline & $P\left(T_{o r}^{(d)} \leq 5\right)$ & 0.6834 & 0.5649 & 0.4587 & 0.4192 & 0.3961 \\
\hline & $P\left(T_{\text {and }}^{(d)} \leq 5\right)$ & 0.3600 & 0.3107 & 0.2152 & 0.1558 & 0.0799 \\
\hline
\end{tabular}



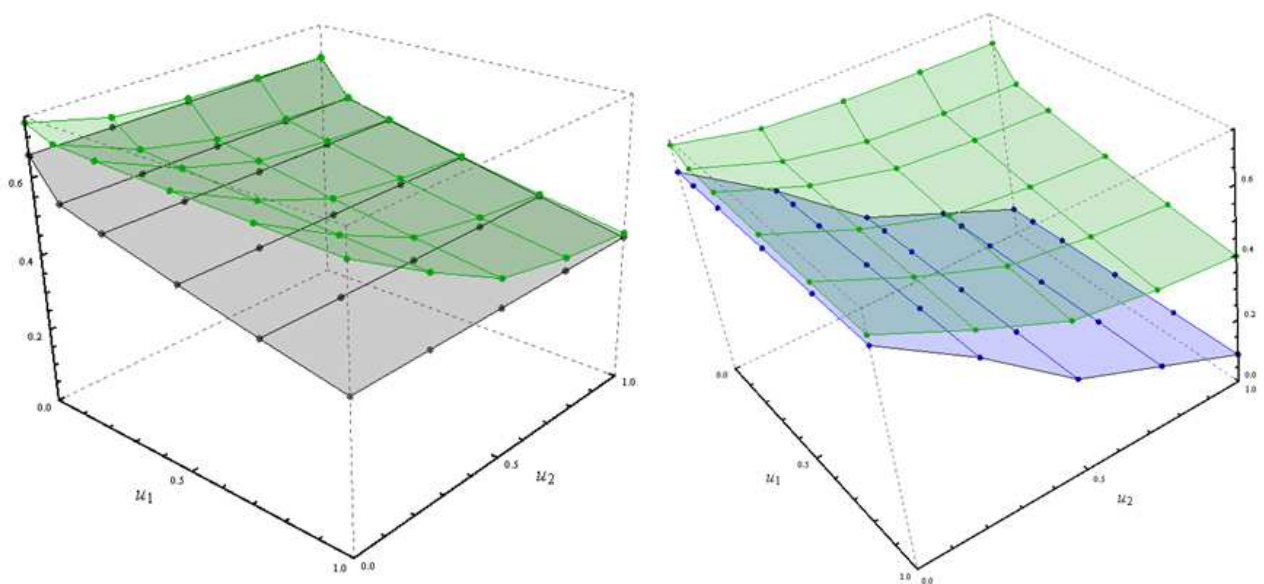

Figure 2: Ruin probabilities until $t=5$ (same parameters): left graph, $P\left(T_{1}^{(d)} \leq 5\right)$ (black) and $P\left(T_{o r}^{(d)} \leq 5\right)$ (green); right graph, $P\left(T_{2}^{(d)} \leq 5\right)$ (blue) and $P\left(T_{o r}^{(d)} \leq 5\right)$ (green).

For Table 2, we computed $P\left[T_{\text {sim }}^{(d)}>t-1, U_{1}(t) \geq 0, U_{2}(t) \geq 0\right]$, i.e. the probability that at least one surplus is non-negative until $t-1$ and both are non-negative at $t$, using Corollary 2.4 and for different initial reserves and time horizons. Of course, these survival probabilities behave as in the cases considered in Table 1.

Table 2: $P\left[T_{\text {sim }}^{(d)}>t-1, U_{1}(t) \geq 0, U_{2}(t) \geq 0\right]$ in function of $t$ and $\left(u_{1}, u_{2}\right)\left(r=0.8, m=1.5, \theta_{1}=0.05, \theta_{2}=0.1\right.$ and $d=0.01)$.

\begin{tabular}{llllll}
\hline$\left(u_{1}, u_{2}\right)$ & $t=1$ & $t=2$ & $t=3$ & $t=4$ & $t=5$ \\
\hline$(0,0)$ & 0.5842 & 0.4568 & 0.4346 & 0.3797 & 0.3420 \\
$(0.1,0.1)$ & 0.6787 & 0.5252 & 0.5022 & 0.4419 & 0.4035 \\
$(0.25,0.25)$ & 0.7112 & 0.6399 & 0.5554 & 0.5010 & 0.4842 \\
$(0.5,0.5)$ & 0.8222 & 0.7242 & 0.6558 & 0.6284 & 0.5897 \\
$(0.75,0.75)$ & 0.8507 & 0.7648 & 0.7214 & 0.6802 & 0.6481 \\
$(1,1)$ & 0.8746 & 0.8069 & 0.7612 & 0.7262 & 0.7025 \\
\hline
\end{tabular}

Effects of reinsurance. We first illustrate possible implications of reinsurance for the insurer. Table 3 gives its ruin probabilities without reinsurance and with the previous reinsurance contract, over different time horizons. For this example, reinsurance is a better option for the insurer except when the reserves $u_{1}$ are null (it is then safer to cover the total risk and keep the whole premium).

Table 3: Insurer's ruin probabilities in function of $t$ and $u_{1}$, without reinsurance $P\left(T^{(d)} \leq t\right)\left(\theta_{1}=0.05, d=0.01\right)$ and with reinsurance $P\left(T_{1}^{(d)} \leq t\right)\left(r=0.8, m=1.5, \theta_{1}=0.05, \theta_{2}=0.1\right.$ and $\left.d=0.01\right)$.

\begin{tabular}{|c|c|c|c|c|c|c|}
\hline \multirow[b]{2}{*}{$u_{1}$} & \multicolumn{2}{|l|}{$t=1$} & \multicolumn{2}{|l|}{$t=2$} & \multicolumn{2}{|l|}{$t=5$} \\
\hline & $P\left(T^{(d)} \leq 1\right)$ & $P\left(T_{1}^{(d)} \leq 1\right)$ & $P\left(T^{(d)} \leq 2\right)$ & $P\left(T_{1}^{(d)} \leq 2\right)$ & $P\left(T^{(d)} \leq 5\right)$ & $P\left(T_{1}^{(d)} \leq 5\right)$ \\
\hline 0 & 0.3451 & 0.4158 & 0.4799 & 0.5143 & 0.6416 & 0.6577 \\
\hline 0.1 & 0.3213 & 0.2353 & 0.4550 & 0.3698 & 0.6204 & 0.5596 \\
\hline 0.25 & 0.2888 & 0.2118 & 0.4198 & 0.3385 & 0.5897 & 0.5269 \\
\hline 0.5 & 0.2421 & 0.1778 & 0.3668 & 0.2920 & 0.5412 & 0.4760 \\
\hline 0.75 & 0.2031 & 0.1493 & 0.3201 & 0.2519 & 0.4958 & 0.4294 \\
\hline 1 & 0.1716 & 0.1254 & 0.2791 & 0.2172 & 0.4535 & 0.3868 \\
\hline
\end{tabular}


The fates of the ceding and reinsurance companies are positively correlated: a large claim is a bad news for both! Let us examine the influence of that positive dependence on the joint survival probability. As proved in Picard et al. (2003a), $P\left(T_{o r}^{(d)}>t\right)$, the probability that both companies are not ruined until $t$, is larger than $P\left(T_{1}^{(d)}>t\right) P\left(T_{2}^{(d)}>t\right)$, the analogous probability calculated as if the cedent and the reinsurer were independent. This is illustrated in Table 4 by measuring the differences of these two probabilities, in absolute and relative values, when $t=5$ and for different $\left(u_{1}, u_{2}\right)$. We observe that the relative differences are here less important when the initial reserves are larger (this is not true in absolute values).

Table 4: Differences $P\left(T_{o r}^{(d)}>5\right)-P\left(T_{1}^{(d)}>5\right) P\left(T_{2}^{(d)}>5\right)$, in absolute values and relative values with respect to the independent case, in function of $\left(u_{1}, u_{2}\right)\left(r=0.8, m=1.5, \theta_{1}=0.05, \theta_{2}=0.1\right.$ and $\left.d=0.01\right)$.

\begin{tabular}{|c|c|c|c|c|c|c|c|c|c|c|}
\hline$u_{1} \backslash u_{2}$ & & 0 & & 25 & & .5 & & 75 & & \\
\hline 0 & 0.1462 & $124.38 \%$ & 0.1425 & $81.42 \%$ & 0.0897 & $36.74 \%$ & 0.0619 & $22.28 \%$ & 0.0301 & $9.67 \%$ \\
\hline 0.1 & 0.1423 & $94.07 \%$ & 0.1524 & $67.67 \%$ & 0.1075 & $34.26 \%$ & 0.0771 & $21.58 \%$ & 0.0382 & $9.53 \%$ \\
\hline 0.25 & 0.1359 & $83.66 \%$ & 0.1476 & $61.02 \%$ & 0.1087 & $32.23 \%$ & 0.0798 & $20.78 \%$ & 0.0403 & $9.34 \%$ \\
\hline 0.5 & 0.1256 & $69.78 \%$ & 0.1392 & $51.96 \%$ & 0.1089 & $29.14 \%$ & 0.0825 & $19.40 \%$ & 0.0429 & $8.98 \%$ \\
\hline 0.75 & 0.1156 & $58.98 \%$ & 0.1305 & $44.72 \%$ & 0.1072 & $26.35 \%$ & 0.0835 & $18.02 \%$ & 0.0446 & $8.57 \%$ \\
\hline 1 & 0.1060 & $50.34 \%$ & 0.1216 & $38.79 \%$ & 0.1041 & $23.82 \%$ & 0.0830 & $16.67 \%$ & 0.0454 & $8.13 \%$ \\
\hline
\end{tabular}

Optimal joint survival. Inspired by e.g. Kaishev and Dimitrova (2006), we consider two optimality problems for maximizing the survival probability of both companies over a fixed horizon. The first question addressed is to find the optimal split of the total premium between the cedent and the reinsurer, i.e. the level $c_{2}$ (or $\theta_{2}$ ) that

$$
\max _{c_{2}} P\left(T_{o r}^{(d)}>t\right) \text {. }
$$

Table 5 gives, for $t=2$, the optimal premium $c_{2}$ and the corresponding joint survival probability in function of $u_{1}$ and $u_{2}$. We note that $c_{2}$ decreases with $u_{2}$ (for fixed $u_{1}$ ), but $c_{2}$ is non-decreasing with $u_{1}$ (for fixed $u_{2}$ ).

Table 5: Optimal $c_{2}$ and associated joint survival probabilities when $t=2$, in function of $\left(u_{1}, u_{2}\right)(r=0.8$, $m=1.5, \theta_{1}=0.05$ and $\left.d=0.01\right)$.

\begin{tabular}{llllll}
\hline$u_{1} \backslash u_{2}$ & & 0 & 0.25 & 0.5 & 0.75 \\
\hline 0 & $c_{2}$ & 0.25 & 0.25 & 0.2 & 0.01 \\
& $P\left(T_{\text {or }}^{(d)}>2\right)$ & 0.463902 & 0.576772 & 0.618062 & 0.654511 \\
\hline 0.25 & $c_{2}$ & 0.37 & 0.36 & 0.2 & 0.01 \\
& $P\left(T_{\text {or }}^{(d)}>2\right)$ & 0.558611 & 0.597721 & 0.659904 & 0.687004 \\
\hline 0.5 & $c_{2}$ & 0.5 & 0.45 & 0.2 & 0.07 \\
& $P\left(T_{\text {or }}^{(d)}>2\right)$ & 0.580630 & 0.646240 & 0.694899 & 0.715798 \\
\hline 0.75 & $c_{2}\left(T_{\text {or }}^{(d)}>2\right)$ & 0.56 & 0.45 & 0.2 & 0.13 \\
& 0.600992 & 0.688630 & 0.724202 & 0.742955 \\
\hline
\end{tabular}

In a similar framework, suppose that the total initial reserves are fixed at a level $u$, and the question now is how to split $u$ into $u_{1}$ and $u_{2}$ for maximizing $P\left(T_{o r}^{(d)}>t\right)$. In Table 6 , we give the optimal split and the corresponding survival probability when $t=2$, for different values $u$. It is worth comparing this probability with $P\left(T^{(d)}>t\right)$, the non-ruin probability for the insurer 
without reinsurance and having $u$ as initial reserves. For this example, $P\left(T^{(d)}>2\right)>P\left(T_{o r}^{(d)}>\right.$ 2 ) in all cases, meaning that reinsurance is not interesting for the insurer if the criterion is to maximize the joint survival probability.

Table 6: Optimal $\left(u_{1}, u_{2}\right)$, joint survival probabilities and survival probabilities without reinsurance when $t=2$, in function of $u\left(r=0.8, m=1.5, \theta_{1}=0.05, \theta_{2}=0.1\right.$, and $\left.d=0.01\right)$.

\begin{tabular}{|c|c|c|c|}
\hline \multirow[t]{2}{*}{$u$} & \multicolumn{2}{|c|}{ Optimal split } & $P\left(T^{(d)}>2\right)$ \\
\hline & $\left(u_{1}, u_{2}\right)$ & $P\left(T_{o r}^{(d)}>2\right)$ & \\
\hline 0 & $(0,0)$ & 0.415458 & 0.520096 \\
\hline 0.2 & $(0.01,0.19)$ & 0.517466 & 0.568782 \\
\hline 0.4 & $(0.01,0.39)$ & 0.597644 & 0.612810 \\
\hline 0.6 & $(0.15,0.45)$ & 0.632158 & 0.652593 \\
\hline 0.8 & $(0.35,0.45)$ & 0.665303 & 0.688507 \\
\hline 1 & $(0.55,0.45)$ & 0.694035 & 0.720896 \\
\hline
\end{tabular}

\subsection{Excess of loss reinsurance}

Let us now consider an excess of loss contract with deductible $r>0$. So, for each claim amount $Z^{(k)}, k \geq 1$, the cedent covers $Z_{1}^{(k)}=\min \left(r, Z^{(k)}\right)$ and the reinsurer $Z_{2}^{(k)}=Z^{(k)}-Z_{1}^{(k)}=$ $\left(Z^{(k)}-r\right)^{+}$. The claim numbers in the successive periods $(t-1, t], t \geq 1$, are i.i.d. random variables, distributed as $N$, independently of the claim sizes. Then, the total claim amounts per period are i.i.d., distributed as $X=\sum_{k=1}^{N} Z^{(k)}$; the parts for the cedent and the reinsurer are $X_{1}=\sum_{k=1}^{N} Z_{1}^{(k)}$ and $X_{2}=\sum_{k=1}^{N} Z_{2}^{(k)}$.

Here too, the companies are assumed to use the expected value principle. Thus, $c_{1}=$ $E(N) E(Z)\left(1+\theta_{1}\right)-c_{2}$, and $c_{2}=E(N) \pi_{Z}(r)\left(1+\theta_{2}\right)$ where $\pi_{Z}(r)$ denotes again the stop-loss premium. If $Z$ is, for instance, exponential with mean $1 / \beta, \pi_{Z}(r)=(1 / \beta) e^{-\beta r}$.

When $Z$ is continuous, it is discretised as before using a span $d$. This yields a random variable $Z^{(d)}$ with values $n d$ and probability masses $q^{(d)}(n), n \in \mathbb{N}$. The corresponding amounts $\left(Z_{1}^{(d)}, Z_{2}^{(d)}\right)$ have values $\left(n_{1} d, n_{2} d\right)$ and probability masses $f^{(d)}\left(n_{1}, n_{2}\right) \equiv P\left(Z_{1}^{(d)}=n_{1} d, Z_{2}^{(d)}=\right.$ $\left.n_{2} d\right)$ given by

$$
f^{(d)}\left(n_{1}, n_{2}\right)= \begin{cases}q^{(d)}\left(n_{1}\right), & \text { for } 0 \leq n_{1} \leq\lfloor r / d\rfloor, n_{2}=0 \\ q^{(d)}\left(\lfloor r / d\rfloor+n_{2}\right), & \text { for } n_{1}=\lfloor r / d\rfloor, n_{2} \geq 1\end{cases}
$$

A convenient situation is when the distribution of $N$ belongs to the Panjer class, i.e. $P(N=$ $n)=(a+b / n) P(N=n-1), n \geq 1$, for some $a, b$. Then, an extended Panjer algorithm allows us to determine the distribution of $\left(X_{1}^{(d)}, X_{2}^{(d)}\right)$ (see Sundt (1999), Section 4 H, and e.g. Mata (2000), Section 3.1). More precisely, the corresponding probability masses $p^{(d)}\left(n_{1}, n_{2}\right) \equiv$ $P\left(X_{1}^{(d)}=n_{1} d, X_{2}^{(d)}=n_{2} d\right)$ can be computed from the recursion: 


$$
\begin{aligned}
& p^{(d)}(0,0)=\sum_{n=0}^{\infty} P(N=n)\left[f^{(d)}(0,0)\right]^{n}, \\
& p^{(d)}\left(n_{1}, 0\right)=\sum_{n=0}^{\min \left(n_{1},\lfloor r / d\rfloor\right)}\left(a+b n / n_{1}\right) q^{(d)}(n) p^{(d)}\left(n_{1}-n, 0\right), \quad \text { for } n_{1} \geq 1, \\
& p^{(d)}\left(n_{1}, n_{2}\right)=\sum_{n=0}^{\lfloor r / d\rfloor-1}\left(a+b n / n_{1}\right) q^{(d)}(n) p^{(d)}\left(n_{1}-n, n_{2}\right)+\left(a+b\lfloor r / d\rfloor / n_{1}\right) \\
& \sum_{n=0}^{n_{2}} q^{(d)}(\lfloor r / d\rfloor+n) p^{(d)}\left(n_{1}-\lfloor r / d\rfloor, n_{2}-n\right), \quad \text { for } n_{1} \geq\lfloor r / d\rfloor+1, n_{2} \geq 1 .
\end{aligned}
$$

In case of a single risk, a different interesting method to approximate compound distributions by discretisation is proposed in Sangüesa (2008).

For illustration, suppose that $N$ is a Poisson variable with parameter 1 and $Z$ is exponentially distributed with mean 1 . In other words, the total claim amount is given by a compound Poisson process. Figure 3 shows the ruin probabilities for at least one company as a function of the deductible $r$, over several time horizons and for different initial reserves. We note that these ruin probabilities decrease here with the deductible $r$. This is also observed for the two marginal ruin probabilities (not given below).
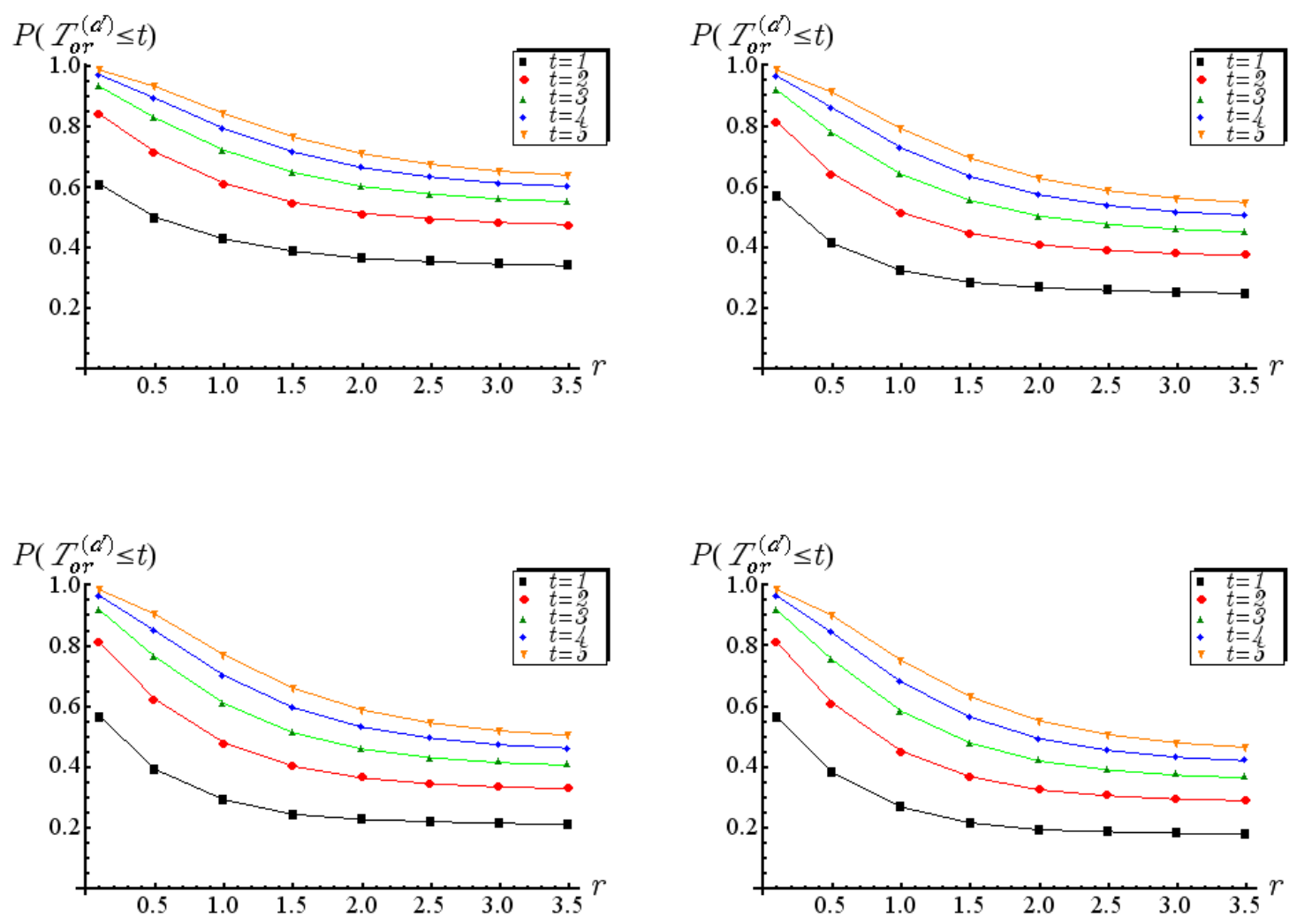

Figure 3: Ruin probabilities for at least one company in function of $r$, for $t=1,2,3,4,5$ and when $\left(u_{1}, u_{2}\right)=(0,0)$ (top, left), $(0.5,0.5)$ (top, right), $(0.75,0.75)$ (bottom, left) and $(1,1)$ (bottom, right) $\left(\theta_{1}=0.05, \theta_{2}=0.1\right.$ and $d=0.01)$. 


\section{Acknowledgements}

The authors thank the Spanish Ministerio de Educación y Ciencia (MICINN) for its support (grant ECO2010-22065-C03-03). Part of this work has been done using the resources at the Centre de Serveis Científics i Acadèmics de Catalunya (CESCA). C. Lefèvre is grateful to the Banque Nationale de Belgique for its support.

\section{References}

Avram, F., Palmowski, Z., Pistorius, M., 2008. A two-dimensional ruin problem on the positive quadrant. Insurance: Mathematics and Economics 42, 227-234.

Bowers, N. L., Gerber, H. U., Hickman, J. C., Jones, D. A., Nesbitt, C. J., 1997. Actuarial Mathematics. The Society of Actuaries, Schaumburg.

Cai, J., Li, H., 2007. Dependence properties and bounds for ruin probabilities in multivariate compound risk models. Journal of Multivariate Analysis 98, 757-773.

Castañer, A., Claramunt, M. M., Gathy, M., Lefèvre, C., Mármol, M., 2011. Ruin problems for a discrete time risk model with non-homogeneous conditions. Scandinavian Actuarial Journal (forthcoming) doi:10.1080/03461238.2010.546144, 1-20.

Centeno, M. L., Simões, O., 2009. Optimal reinsurance. Revista de la Real Academia de Ciencias Exactas Fisicas y Naturales Serie A-Matematicas 103, 387-404.

Chan, W.-S., Yang, H., Zhang, L., 2003. Some results on ruin probabilities in a two-dimensional risk model. Insurance: Mathematics and Economics 32, 345-358.

Denuit, M., Frostig, E., Levikson, B., 2007. Supermodular comparison of time-to-ruin random vectors. Methodology and Computing in Applied Probability 9, 41-54.

Denuit, M., Lefèvre, C., Picard, P., 2003. Polynomial structures in order statistics distributions. Journal of Statistical Planning and Inference 113, 151-178.

Dimitrova, D. S., Kaishev, V. K., August 2010. Optimal joint survival reinsurance: An efficient frontier approach. Insurance: Mathematics and Economics 47, 27-35.

Gong, L., Badescu, A. L., Cheung, E. C., 2012. Recursive methods for a multi-dimensional risk process with common shocks. Insurance: Mathematics and Economics 50, 109-120.

Goovaerts, M. J., Kaas, R., Van Heerwaarden, A. E., Bauwelinckx, T., 1990. Effective Actuarial Methods. North-Holland, Amsterdam.

Guo, J. Y., Yuen, K. C., Zhou, M., 2007. Ruin probabilities in Cox risk models with two dependent classes of business. Acta Mathematica Sinica-English Series 23, 1281-1288.

Ignatov, Z. G., Kaishev, V. K., 2000. Two-sided bounds for the finite time probability of ruin. Scandinavian Actuarial Journal 1, 46-62.

Ignatov, Z. G., Kaishev, V. K., 2004. A finite-time ruin probability formula for continuous claim severities. Journal of Applied Probability 41, 570-578.

Kaas, R., 1993. How to (and how not to) compute stop-loss premiums in practice. Insurance: Mathematics and Economics 13, 241-254. 
Kaas, R., Goovaerts, M. J., Dhaene, J., Denuit, M., 2008. Modern Actuarial Risk Theory: Using R. Springer, Heidelberg.

Kaishev, V. K., Dimitrova, D. S., December 2006. Excess of loss reinsurance under joint survival optimality. Insurance: Mathematics and Economics 39, 376-389.

Kaishev, V. K., Dimitrova, D. S., Ignatov, Z. G., 2008. Operational risk and insurance: a ruin-probabilistic reserving approach. The Journal of Operational Risk 3, 39-60.

Kaz'min, Y. A., 2002. Encyclopaedia of Mathematics. Springer, New York, Ch. Appell polynomials.

Lefèvre, C., Loisel, S., 2009. Finite-time ruin probabilities for discrete, possibly dependent, claim severities. Methodol. Comput. Appl. Probab. 11, 425-441.

Lefèvre, C., Picard, P., 2006. A nonhomogeneous risk model for insurance. Computers and Mathematics with Applications 51, 325-334.

Lefèvre, C., Picard, P., 2011. A new look at the homogeneous risk model. Insurance: Mathematics and Economics 49, 512-519.

Li, J., Liu, Z., Tang, Q., 2007. On the ruin probabilities of a bidimensional perturbed risk model. Insurance: Mathematics and Economics 41, 185-195.

Li, S., Lu, Y., Garrido, J., 2009. A review of discrete-time risk models. Revista de la Real Academia de Ciencias Exactas Fisicas y Naturales Serie A-Matematicas 103, 321-337.

Mata, A. J., 2000. Pricing excess of loss reinsurance with reinstatements. Astin Bulletin 30, $349-368$.

Niederhausen, H., 1988. Encyclopedia of Statistical Sciences. Wiley, New York, Ch. Sheffer polynomials.

Picard, P., Lefèvre, C., 1996. First crossing of basic counting processes with lower non-linear boundaries: a unified approach through pseudopolynomials (i). Advances in Applied Probability $28,853-876$.

Picard, P., Lefèvre, C., 1997. The probability of ruin in finite time with discrete claim size distribution. Scandinavian Actuarial Journal 1, 58-69.

Picard, P., Lefèvre, C., Coulibaly, I., 2003a. Multirisks model and finite-time ruin probabilities. Methodology and Computing in Applied Probability 5, 337-353.

Picard, P., Lefèvre, C., Coulibaly, I., 2003b. Problèmes de ruine en théorie du risque à temps discret avec horizon fini. Journal of Applied Probability 40, 527-542.

Rytgaard, M., 2004. Encyclopedia of Actuarial Science. Wiley, Chichester, Ch. Stop-loss reinsurance.

Sangüesa, C., 2008. Error bounds in approximations of random sums using gamma-type operators. Insurance: Mathematics and Economics 42, 484-491.

Sundt, B., 1999. On multivariate Panjer recursions. Astin Bulletin 29, 29-46. 